\title{
Mujer y academia en tiempos de pandemia COVID 19
}

\section{Women and scholarship in COVID 19 pandemic}

\author{
Solange Rivera Mercado
}

Palabras clave: mujer; género; academia; COVID-19.

Keywords: woman; gender; academy; COVID-19.

Hace años existe, a nivel nacional e internacional, una preocupación por abordar las brechas de género en lo social y educativo. Chile es un país particularmente afectado por estas brechas en detrimento de las mujeres, ocupando el lugar 63 de 144 naciones en el Índice de brechas de género del Foro Económico Mundial (Foro Económico mundial, 2017). Brecha de género en el mundo. Informe del Foro Económico Mundial . Los resultados que muestran un mayor rezago para nuestro país, según lo evaluado por este índice, fueron los indicadores de "participación económica y oportunidades". Esta área muestra una baja participación de las mujeres en el mercado laboral, disparidad en las remuneraciones, escasa diversidad en los campos de ocupación y la falta de presencia de mujeres en cargos altos en empresas privadas u organizaciones públicas.

Actualmente en Chile, según cifras del Ministerio de Educación, la participación de mujeres entre sujetos titulados de carreras de pregrado se mantuvo en alrededor de 56,6 \% entre los años 2005 y 2018. En el mismo período, se evidenció un aumento importante en la participación de las mujeres en las titulaciones de posgrado, de 36,4\% en 2005 a 50\% en 2018 (CONICYT, 2018). A pesar de estos avances, se mantienen importantes brechas en la progresión y participación de las mujeres en la academia, siendo pocas las que llegan a ocupar puestos de alta responsabilidad y toma de decisiones.

En el área de la medicina, estudios recientes reportan la dificultad que tienen las mujeres para poder optar a posiciones académicas importantes, e incluso recalcan la diferencia de ingresos que existe entre hombres y mujeres por realizar el mismo trabajo (Jena et al., 2016). Este fenómeno conocido como «segregación vertical» hace alusión a la sub representación de la mujer en puestos de mayor jerarquía académica (Tesch \& Nattinger, 1997) y se relaciona a una menor promoción y asignación de recursos institucionales en las mujeres (Clark, 2005).

Se ha descrito en la literatura un «sesgo silencioso» también conocido como "techo de cristal" que se aplica en las mujeres dedicadas a la medicina y las ciencias biomédicas que buscan progresión académica e identifica las barreras implícitas, individuales e institucionales que explican la desigualdad de género (Pingleton et al., 2016).

En la investigación el panorama también es desigual. Las causas de esta situación son multifactoriales y apuntan entre otros aspectos a la dificultad para conciliar trabajo y vida personal en las mujeres (Makarem \& Wang, 2020). Un estudio transversal en un grupo de investigadores norteamericanos (Jolly et al., 2014), reportó que las investigadoras médicas casadas o en pareja con hijos, informaban una dedicación de 8,5 horas más a la semana para la crianza de los hijos o las actividades domésticas que sus homólogos masculinos. Además, el tiempo dedicado a las actividades de investigación fue menor en estas mujeres. Esto sugiere que el tiempo otorgado al trabajo doméstico compite con el tiempo de trabajo académico y que la actividad específica con la que compite es la investigación. De hecho, el tiempo de investigación puede ser el aspecto más flexible de una carrera académica médica y, por lo tanto, el más susceptible de transar cuando existen demandas en competencia. Lamentablemente, también es la actividad más crítica para el éxito académico. Por lo tanto, si el tiempo dedicado al trabajo doméstico desplaza el tiempo de investigación, puede ser un mecanismo por el cual persistan las diferencias de género en el éxito académico de personas igualmente aptas y motivadas.

Esto se ve reflejado en el número de publicaciones científicas

Departamento de Medicina Familiar, Facultad de Medicina, Pontificia Universidad Católica de Chile. Autor de correspondencia: strivera@uc.cl 
lideradas por mujeres. Existen diversos estudios para las distintas disciplinas de la medicina (Hart et al., 2019; Vranas et al., 2019; Brown et al., 2020) (que concuerdan en mostrar que las mujeres representan menos de un tercio de los primeros autores de artículos de investigación, con un leve aumento durante la última década, aumentando las probabilidades de ser coautora si el autor principal es también una mujer (Whitelaw et al., 2020). En general, las cifras de representación femenina no superan el $41 \%$ de los autores de los articulos médicos en la era pre pandemia (Gavet-Ageron et al.,2019).

Ahora, con más de 12 meses en estado de emergencia por la pandemia COVID-19, muchos gobiernos han cerrado escuelas e implementado requisitos de distanciamiento social que limitan las opciones de cuidado infantil, al mismo tiempo que exigen que los académicos e investigadores trabajen desde sus hogares. Es de esperar que estas restricciones tengan un impacto desproporcionado en la vida académica de las mujeres, en comparación con los hombres (Viglione, 2020). Sin dejar de mencionar que más del $70 \%$ de la fuerza laboral en salud son mujeres (OEA, 2020). Estamos conociendo recién del impacto que podrían tener estas nuevas demandas de servicios profesionales y cómo han influido en la productividad académica en nuestras colegas.

El aumento de las responsabilidades del cuidado infantil, la disminución de las oportunidades de investigación y desarrollo profesional, son motivo de preocupación para el avance en términos de equidad de género (Gabster et al., 2020). La búsqueda de soluciones comienza con la identificación de problemas, por lo que se hace necesario monitorear cómo les está yendo a las mujeres en la medicina en estos tiempos. Explorando el impacto de COVID-19 en las mujeres en la medicina y la ciencia, analizando distintos dominios, incluida su salud mental.

Los primeros datos muestran que COVID-19 ha afectado significativamente las publicaciones de mujeres. Andersen y colegas compararon la autoría de 1179 artículos médicos COVID-19 con 37531 artículos de las mismas revistas en 2019. La participación de las mujeres en la autoría general, primera autoría y última autoría de los artículos COVID-19 disminuyó de un 30\%, 28\% y 22\% a un $16 \%, 23 \%$ y $16 \%$, respectivamente.

En diciembre del 2020, la Comisión de Medicina y Mujer de la Sociedad de Medicina Interna General, publicó sugerencias para proteger a las mujeres de las consecuencias del COVID-19 (Narayana et al., 2020). Estas recomendaciones coinciden con los resultados de una reciente revisión de la literatura (Segovia-Saiz et al., 2019) que identificó los obstáculos y factores potencialmente favorecedores para el avance de la carrera de las mujeres académicas e investigadoras en ciencias biomédicas, algunos de estos son 1) que haya ejemplos de mujeres científicas en puestos de liderazgo; 2) mentorización; 3) favorecer la conciliación hogar-trabajo; 4) transparencia en la contratación; 5) participación de las mujeres en la toma de decisiones 6) realizar auditorías de género en la evaluación de la investigación; 7) conciencia de las desigualdades de género en las instituciones; 8) promover la colaboración; y 9) la equidad salarial.

Para contrarrestar los posibles desequilibrios de género, se propone a las instituciones reevaluar cómo recompensan el esfuerzo académico y organizacional en tiempos de pandemia. Algunos autores recomiendan, incluir el trabajo de servicio relacionado con la pandemia en sus currículos vitae y evaluaciones de desempeño (Gabster et al., 2020).

Los desafíos que enfrentan las mujeres en el mundo académico están bien documentados en épocas no pandémicas. Asegurar que el rendimiento académico de las mujeres no se vea afectado de manera desproporcionada por COVID-19 podría salvaguardar las trayectorias profesionales de éstas.

La ciencia y la innovación se benefician de la diversidad. Tal y como Segovia et al. mencionan, el primer paso para avanzar en términos de equidad de género en ciencias biomédicas, es reconocer que existe un techo de cristal y que esta falta de equidad sigue siendo una amenaza y una desventaja competitiva en investigación que entorpece el avance de la generación actual de mujeres académicas e investigadoras, subutiliza su talento y potencial, desaprovechando un considerable capital humano. Hay mucho por hacer y podemos hacerlo mejor.

\section{Referencias}

Andersen JP, Nielsen MW, Simone NL, Lewiss RE. \& Jagsi R. (2020). COVID-19 medical papers have fewer women first authors than expected. elife 9, e58807

Brown MA, Erdman MK, Munger AM. \& Miller AN. (2020) Despite Growing Number of Women Surgeons, Authorship Gender Disparity in Orthopaedic Literature Persists Over 30 Years. Clin Orthop Relat Res. 478, 1542-1552.

Clark Blickenstaff J. (2005). Women and science careers: leaky pipeline or gender filter?. Gender and education 17, 369-386.

Comisión Nacional de Investigación Cientifica y Tecnológica (CONICYT) 2018. Reporte de participación femenina 2018 (periodo 2009-2018). Accedido en https://www.conicyt.cl/wp-content/ uploads/2015/03/REPORTE-DE-GENERO-2018.pdf el 20 de marzo de 2021. 
Foro Económico mundial 2017. Brecha de género en el mundo. Informe del Foro Económico Mundial. Accedido en https://www. weforum.org/reports/the-global-gender-gap-report-2017 el 22 de marzo de 2021.

Gabster BP, van DaalenK, Dhatt, R. \& Barry M. (2020). Challenges for the female academic during the COVID-19 pandemic. Lancet 395, 1968-1970.

Gayet-Ageron A, Poncet A, Perneger T. (2019) Comparison of the contributions of female and male authors to medical research in 2000 and 2015: a cross-sectional study. BMJ Open 9,e024436.

Hart KL, Frangou S. \& Perlis RH. (2019) Gender Trends in Authorship in Psychiatry Journals From 2008 to 2018. Biol Psychiatry. 15;86, 639-646.

Jena AB, Olenski AR. \& Blumenthal DM. (2016). Sex Differences in Physician Salary in US Public Medical Schools. JAMA Intern Med.

176, 1294-1304.

Jolly S, Griffith KA, DeCastro R, Stewart A, Ubel P. \& Jagsi R. (2014). Gender differences in time spent on parenting and domestic responsibilities by high-achieving young physician-researchers. Annals of internal medicine 160, 344-353.

Makarem Y. \& Wang J. (2020). Career experiences of women in science, technology, engineering, and mathematics fields: $A$ systematic literature review. Human Resource Development Quarterly 31, 91-111.

Narayana S, Roy B, Merriam S, Yecies E, Lee RS, Mitchell JL, Gottlieb AS; , on behalf of the Society of General Internal Medicine's Women and Medicine Commission (2020). Minding the Gap: Organizational Strategies to Promote Gender Equity in Academic Medicine During the COVID-19 Pandemic. J Gen Intern Med. 35, 3681-3684.
OEA, CIM. (2020). COVID-19 en la vida de las mujeres. Razones para reconocer los impactos diferenciados Comisión Interamericana de Mujeres. p.25.

Pingleton SK, Jones EV, Rosolowski TA. \& Zimmerman MK. (2016) Silent Bias: Challenges, Obstacles, and Strategies for Leadership Development in Academic Medicine-Lessons From Oral Histories of Women Professors at the University of Kansas. Acad Med. 91:1151-7.

Segovia-Saiz C, Briones Vozmediano E, Pastells Peiró R, González-María E. \& Gea Sánchez M. (2019). Techo de cristal y desigualdades de género en la carrera profesional de las mujeres académicas e investigadoras en ciencias biomédicas. Gaceta Sanitaria 34, 403-410.

Tesch BJ. \& Nattinger AB. (1997). Career advancement and gender in academic medicine. Journal-Irish Colleges of Physicians and Surgeons 26, 172-176.

Viglione G. (2020) Are women publishing less during the pandemic? Here's what the data say. Nature. 581, 365-366.

Vranas KC, Ouyang D, Lin AL, Slatore CG, Sullivan DR, Kerlin MP, Liu KD, Baron RM, Calfee CS, Ware LB, Halpern SD, Matthay MA, Herridge MS, Mehta S \& Rogers AJ. (2020) Gender Differences in Authorship of Critical Care Literature. Am J Respir Crit Care Med. 201, 840-847.

Whitelaw S, Thabane L, Mamas MA, Reza N, Breathett K, Douglas PS. \& Van Spall HGC. (2020) Characteristics of Heart Failure Trials Associated With Under-Representation of Women as Lead Authors. J Am Coll Cardiol. 2020 Oct 27, 76,1919-1930. 